\title{
Deporte y competencias genéricas en la universidad: diseño y validación del "competest".
}

\section{Sports and generic skills at university: the design and validation of the "competest".}

\author{
M. Mónica ÁLVAREZ BENÍTEZ ${ }^{1}$, I. Inmaculada ASENSIO MUÑOZ \\ y José Manuel GARCÍA RAMOS. \\ Universidad Complutense de Madrid
}

Recibido: Octubre 2012

Aceptado: Diciembre 2012

\begin{abstract}
Resumen: El competest es un instrumento diseñado para medir, mediante autoinforme, determinadas competencias genéricas útiles para el desempeño profesional de los universitarios, en el marco de una investigación más amplia que pretende obtener evidencia en la que apoyarse para maximizar el potencial formativo del deporte en la universidad actual, a la que se le demanda una función profesionalizante cada vez más exigente. El objetivo concreto de este artículo es el estudio métrico del cuestionario, que parte de la descripción del proceso seguido para el diseño y validación de contenido del competest y resume el trabajo empírico, realizado a partir de su aplicación piloto a una muestra de 288 universitarios españoles y mexicanos, con una edad media de 22 años, en la que el $35,8 \%$ son varones y el 64,2\% mujeres, y en la que el $80,2 \%$ practica deporte. Los resultados aportan evidencia acerca de la fiabilidad del cuestionario $(\alpha=0.958)$ y la fiabilidad y validez concurrente de sus ítems, así como de su utilidad para evaluar el impacto en la autopercepción competencial de la práctica deportiva.
\end{abstract}

Palabras clave: Deporte, Educación Superior, Competencias, Fiabilidad, Análisis de items.

\begin{abstract}
The competest is an instrument which has been designed to measure specific generic skills, by means of a self-evaluation, which are useful for the professional development of university students. This test is set within the framework of broader research aimed at collecting evidence to support maximizing the educational potential of sports in present-day university settings, thereby responding to an ever increasing demand for a development in professionalism within the university. The main objective of this article is the metric study of the questionnaire, which is based on a description of the process followed by the design and validation of the content of the competest and a summary of the empirical study done after carrying out a pilot test done with a sampling of 288 Spanish and Mexican university students and graduates, whose average age is 22 , with $35.8 \%$ males and $64.2 \%$ females, together with a
\end{abstract}

\footnotetext{
${ }^{1}$ Becada por el CONACYT para la realización de la Tesis Doctoral de la que este trabajo forma parte..
} 
high percentage of participants active in sports $(80.2 \%)$. The results show evidence towards the reliability of the questionnaire $(\alpha=0.958)$ and the concurrent reliability and validity of its items, as well as its usefulness for evaluating the impact in the self-perception of one's abilities of the practice of sports.

Key words: Sport, Higher Education, Skills, Reliability, Item analysis.

Aunque las funciones de la universidad han cambiado a lo largo de su historia, son variadas y no existe pleno consenso acerca de cuáles son o deben ser prioritarias (Orden et al., 2002), lo cierto es que su función profesionalizante es una de las que menos se discute. A nivel internacional, está claro que entre los grandes fines de la universidad está la formación de profesionales con un alto nivel de cualificación. En Europa, con la convergencia en materia de educación superior, esta función ha cobrado protagonismo y la Declaración de Bolonia supone el punto de arranque de un proceso en el que las universidades que conforman el Espacio Europeo de Educación Superior han acordado conjugar esfuerzos para trabajar, entre otras cosas, por una formación en aras de la óptima empleabilidad de sus ciudadanos. En el Tratado de Lovaina (Conference of European Ministers Responsible for Higher Education, 2009), la Declaración de Budapest-Vienna (Minister Responsible For The European Higher Education Area, 2010) o en el informe de Tendencias 2010 emitido por la European University Association (2010) se encuentran las propuestas más actuales y las líneas de trabajo específicas. Entre los ejes vertebradores de los acuerdos surgidos del espíritu de Bolonia, está el impulso a la sociedad del conocimiento, como motor para una economía más dinámica, y la participación directa de las universidades en la formación de graduados calificados que respondan adecuadamente a las demandas tradicionales y emergentes de cada profesión en los diferentes escenarios en que ésta tenga que desarrollarse.

Estos planteamientos han llevado a destacar el papel de las universidades en el desarrollo de las competencias que los estudiantes deben adquirir para un adecuado ejercicio profesional, así como para resolver los problemas de diversa índole que pueden surgir según los diferentes niveles de ocupación. En un marco así, el proyecto Tuning (González \& Waguenaar, 2006) ha tenido una enorme importancia por su carácter pionero, por el gran impacto de su metodología y por su efecto aglutinador de universidades (Méndez, 2009) no sólo en Europa, sino también en América Latina (Beneitone, 2007, 2008; González, Waguenaar \& Beneitone, 2004).

Desde una perspectiva pedagógica, el concepto de competencia carece de una definición unívoca y clara (Orden, 2011) y pueden referirse al saber, al saber hacer y al ser (Delors et al., 1998). Algunos autores hacen hincapié en el dominio del saber, en el desarrollo intelectual, y afirman que las competencias se encuentran necesariamente vinculadas al conocimiento, ya que se refieren a la aptitud, al dominio de habilidades y destrezas, pero con un ingrediente esencial que es la capacidad de la persona para seleccionar y movilizar los conocimientos y recursos adquiridos, haciendo uso efectivo de estas herramientas en el momento justo y buscando el éxito en la tarea (Cano, 2005). Para la mayoría, sin embargo, las competencias se manifiestan principalmente 
por la conducta lograda y se califican en función al comportamiento que se tiene en un determinado puesto de trabajo, ante una determinada tarea; este comportamiento se manifiesta en diferentes niveles de dominio y estrictamente debe evaluarse mediante la acción (Asis, 2007; Pereda \& Berrocal, 2001; OIT \& CINTERFORD, 2010). Pero, la realidad es que la formación en competencias profesionales en la universidad actual se concibe como una tarea compleja que va más allá de la mera posesión de conocimientos y habilidades propias de una profesión, incluso más allá del saber hacer en circunstancias vinculadas a escenarios laborales concretos, ya que abarca la capacidad del profesional para adaptarse, para innovar, para responder a las demandas clásicas y emergentes con responsabilidad, autonomía y con "compromiso social" (González \& González, 2008, 187). El llegar a ser competente en una profesión implica conocimientos teóricos y prácticos, pero también actitudes ante uno mismo, ante los demás y ante la profesión; supone la integración de elementos cognitivos y emocionales que lleven a la elección y puesta en marcha del protocolo de actuación más adecuado en cada caso y a un desempeño ético y de calidad, funcional, eficaz y eficiente. Es evidente que el repertorio concreto de competencias depende de la profesión de la que se trate, pero es posible identificar algunas más genéricas y trasversales que pueden ser requeridas por un elenco importante de profesiones y que de manera muy directa se han trabajado habitual y específicamente en muchos deportes, sobre todo a nivel de competición.

La formación por competencias así concebida exige el desarrollo en paralelo de metodologías, técnicas e instrumentos de evaluación de las mismas que permitan a la universidad la constatación del nivel de logro alcanzado por los estudiantes, tanto con fines formativos o de mejora, no sólo del aprendizaje sino también de los procesos educativos, como con finalidad sumativa, de calificación o de certificación de resultados de aprendizaje. La complejidad teórica que rodea al término "competencia" se refleja necesariamente en la dificultad técnica para el desarrollo de procedimientos de evaluación suficientemente discriminativos, válidos, fiables y que aporten una medida estandarizada que asegure la comparabilidad de las puntuaciones entre estudiantes y en el tiempo (Castro, 2011). Sin duda, las limitaciones actualmente existentes en la calidad de las medidas de las competencias, especialmente cuando éstas transcienden el ámbito del saber adquirido por los estudiantes, repercute en el uso evaluativo que se pueda hacer de las mismas. La dificultad de evaluación ha dado lugar al desarrollo de proyectos de innovación sobre esta temática que intentan aportar herramientas diversas y optimizar el uso de las plataformas virtuales con este fin. Algunas aportaciones interesantes de e-evaluación son el proyecto evalcomix (Ibarra, 2008) o el proyecto forcom, cuyos principales resultados se resumen en Álvarez (2011).

Por otro lado, se está trabajando en la elaboración instrumentos desde la perspectiva de la autoevaluación. Se trata de cuestionarios, inventarios o tests que por lo general se refieren a competencias genéricas, difícilmente evaluables con los medios tradicionales. Las competencias específicas son disciplinares, son acciones de dominio propias del área temática de un campo de estudio concreto (Rodríguez \& Vieira, 2009), y su evaluación, al estar vinculada al área de conocimiento, a la materia o a la 
asignatura, puede hacerse más fácilmente mediante un proceso con predominio del enfoque hetero-evaluativo, en el que los profesores o los equipos docentes se basan, para la emisión del juicio evaluativo, en resultados de pruebas objetivas, de exámenes de lápiz y papel o en trabajos, memorias, proyectos realizados por los estudiantes o en pruebas de ejecución. Sin embargo, las competencias genéricas se relacionan más con actitudes y comportamientos comunes a diferentes áreas disciplinares o a distintos ámbitos académicos, laborales o de producción. Pueden ser, por ejemplo, la capacidad de trabajar en equipo, las habilidades para la negociación, para la planificación, etc. (Mertens, 1997). En este sentido, la competencias genéricas se encuentran implícitas en la epistemología de la materia o en la esencia de la actividad y no necesariamente se han de hacer siempre explícitas (Jones, 2006). Además, por lo general no existe un lugar, un contenido concreto y un espacio determinado destinado a ese aprendizaje; más bien se trata de un cometido común, en el que convergen diferentes materias, algo inherentemente unido al carácter mismo de la disciplina o del campo de actuación del que se trata, de ahí que las competencias genéricas excedan los límites de la asignatura y se encuentren perfiladas muchas veces en las políticas institucionales, en la cultura de la universidad o de la facultad o de la profesión, en la transversalidad de los curricula o en las prácticas internas, externas y practicum. Su carácter genérico hace necesario que estas competencias se hayan de trabajar intencionalmente de manera colegiada por parte del profesorado, pero muchas veces este carácter global y extra-curricular aconseja que se aborden de manera integral, más allá del aula, en situaciones como las que surgen, por ejemplo, de las actividades culturales, sociales y deportivas que se desarrollan en la institución y fuera de ella. En cualquier caso, la adquisición y nivel de dominio de las competencias genéricas ha de ser también objeto de evaluación, en la medida en que son relevantes para el desempeño profesional de calidad, pero el enfoque evaluativo a adoptar requiere a menudo de los autoinformes del estudiante, que pueden verse complementados por la evaluación externa, según la función específica que en cada momento dicha evaluación tenga que cumplir.

Muchas veces las competencias genéricas que interesa evaluar son competencias emocionales, ya que se refieren a cualidades personales que reflejan los valores y creencias culturales trasformados por un significado interno, por lo que pueden definirse y medirse a través de las aportaciones al respecto que se han hecho desde la investigación sobre inteligencia emocional. La inteligencia emocional se entiende como un complemento a la inteligencia racional, necesario especialmente para el desarrollo de actividades complejas como las que se plantean a menudo en el ejercicio profesional para el que preparan las carreras universitarias. Las respuestas emocionales de las personas están en relación directa con su autoconocimiento, su autoconfianza y su autocontrol pero tienen indudablemente una dimensión social, y dependen también del dominio de las normas sociales y del manejo que se tiene de las relaciones con los otros (Goleman, 2007; Saarni, 2000; Salovey \& Mayer, 1990).

La definición de la competencia emocional, como afirma Saarni (2000), se encuentra en continua construcción, pero se puede perfilar "como la demostración de la autoeficacia del impulso emocional en el contexto social, entendiendo por autoeficacia las creencias individuales que las personas poseen acerca de sus posibilidades para 
lograr el resultado deseado" (Saarni, 2000, p. 68). Este autor presenta la explicación de los factores que intervienen la competencia emocional, entre los que es posible observar una constante que es la toma de conciencia y aceptación de los estados de la emoción, expresados en correcta sintonía con el contexto cultural y los valores morales de los que la persona se ha apropiado, característica de una competencia emocional madura (Bisquerra \& Pérez, 2007). El desarrollo de la competencia emocional implica, pues, un proceso complejo que abarca la conciencia emocional, la regulación emocional, la autonomía emocional, la competencia social y la competencia para la vida y el bienestar.

Una formación integral que prepara al sujeto para la vida (Bisquerra, 2003; Bisquerra \& Pérez, 2007) no puede dejar al margen el desarrollo de las competencias emocionales cuyo impacto afecta a múltiples planos. En el plano familiar y de salud, la educación emocional contribuirá a organizar la vida de forma sana y equilibrada, facilitando experiencias de satisfacción y bienestar (Bisquerra \& Pérez, 2007; Saarni, 2000). Y, en el plano laboral, que es el que más nos interesa en este estudio, contribuirá al desarrollo de la asertividad, de la regulación, de la empatía, de la motivación, del trabajo en equipo, de la capacidad de resolución de conflictos y ayudará a enfrentar con sentido positivo los retos que se planteen (Repetto et al. 2007; Weisinger, 1998) desde la empresa o en el puesto de trabajo que se desempeñe en cada caso.

Por otra parte, existen competencias que, sin ser emocionales, van más allá del saber e incluso del saber hacer, afectando al ser, y se requieren cada vez más desde el mundo laboral. Se reclama, en mayor o menor medida, según el puesto, capacidad de liderazgo, empuje, empowerment, pensamiento estratégico, visión de futuro y detección de oportunidades, comunicación verbal y no verbal, trabajo en equipo, eficacia y eficiencia. Se trata, en este caso, de competencias directivas o gerenciales, más vinculadas a puestos de gestión, pero muy relacionadas con el éxito de cualquier actividad orientada a resultados, por lo que se plantean como competencias comunes a múltiples campos disciplinares y laborales. Otras competencias laborales de gran demanda en la sociedad actual tienen que ver con la disciplina, el cumplimiento y compromiso personal, los valores y la actitud ética, la capacidad de innovación, el espíritu emprendedor y responsabilidad en la asunción de riesgos, el deseo de crecimiento y mejora, la apertura a la evaluación y al aprendizaje continuo, empeño, perseverancia o resistencia a la frustración. Qué competencias genéricas incluir en un cuestionario con fines evaluativos y cuáles no resulta por lo general una decisión compleja. En un estudio al respecto, Palmer et al. (2009), partieron de un listado de 31 competencias genéricas y concluyeron que la importancia otorgada por las diferentes audiencias estudiadas (empleadores y académicos) no coincidía por lo general.

La elección de competencias finalmente incluidas en nuestro estudio se ha hecho principalmente considerando como factor clave su transversalidad y su potencial adquisición y desarrollo a través de la práctica deportiva, lo que puede contribuir a la consolidación y configuración pedagógica del deporte como actividad formativa en la universidad. En esta línea, Mayocchi y Hanrahan (2007) aportan alguna evidencia cuando en su estudio identifican competencias ganadas por la práctica habitual de deporte y la tensión vivida en la competición que pueden transferirse al trabajo, entre 
las que se encuentran la capacidad de rendir bajo presión, las habilidades de resolución de problemas, la capacidad de organización, el cumplimiento de plazos, la respuesta a los desafíos, el reconocimiento de las propias limitaciones, la adaptabilidad, la dedicación o la perseverancia. En el plano administrativo, los deportistas desarrollan competencias como las habilidades para apreciar las diferencias individuales, la seguridad de sí mismos manifestada en una conducta asertiva, un espíritu competitivo y la orientación a la meta. En especial, los deportistas que desempeñan el papel de capitán de su equipo desarrollan estrategias de supervisión y liderazgo, identificando claramente hasta dónde pueden empujar a la gente y qué les motiva.

Para la medida del tipo de competencias de que aquí se trata hay actualmente en España algunas adaptaciones de pruebas elaboradoras en otros contextos, como las que recogen Fernández Berrocal et al. (2009) para competencias emocionales o algunas aportaciones más específicas, como la de Solanes et al. (2008), que construyen y validan un cuestionario de 45 preguntas para la evaluación de competencias genéricas en la universidad; la de Arruza et al. (2005) que elaboran y validan un cuestionario para la evaluación de competencias emocionales en deportistas; la de Iglesias (2009) para la evaluación de competencias emocionales en universitarios; la de MartínezRodríguez \& Carmona (2010) que, desde otra perspectiva, aportan un test de factores socio-personales para la inserción laboral de los jóvenes. El instrumento que aquí se presenta, el competest, es un inventario diseñado como herramienta de autoevaluación de la adquisición de determinadas competencias genéricas, emocionales, directivas y laborales que pueden resultar útiles para el desempeño profesional de los graduados universitarios. Como fundamento teórico, se basa en el tratamiento y definición que hacen Villa y Poblete (2007) de las competencias genéricas y en las competencias emocionales descritas por Salovey y Mayer (1990) y dadas a conocer a nivel mundial por Daniel Goleman (2007). El instrumento se centra en las competencias que las empresas solicitan a los demandantes de puestos directivos y en otras competencias profesionales valoradas en cualquier puesto (Alles, 2002, 2006; Blanco, 2007; Boyatzis, 1982; Cubeiro \& Gallardo, 2008; Stogh, 2009). Su peculiaridad estriba en que el conjunto de competencias a que se refiere se ha seleccionado porque se presupone su relación con el ejercicio del deporte como actividad transversal, fomentado y practicado en la universidad independientemente de la titulación de la que se trate. La puesta a prueba de la hipótesis acerca de la relación entre la práctica del deporte a diferente nivel y el desarrollo de determinadas competencias es objeto de un trabajo de investigación más amplio, del que éste resulta ser un preliminar cuyo objetivo principal es el estudio de las características técnicas del instrumento (fiabilidad y validez de contenido) y de los elementos que lo componen (índices de homogeneidad, de validez concurrente e impacto de los ítems).

\section{Método}

Para el diseño del instrumento se partió de un planteamiento axiomático consistente en la definición de un marco teórico de las competencias que se pretendía evaluar, a partir del cual se obtuvieron en primer lugar las dimensiones, subdimensiones e indicadores de los que surgieron posteriormente los ítems o cuestiones. La versión 
inicial del inventario se sometió a un proceso de validación de contenido, mediante juicio de expertos, del que se obtuvo una versión definitiva que se somete a estudio empírico, con el fin de obtener evidencia acerca de su fiabilidad y de la homogeneidad, capacidad discriminativa, validez e impacto de los ítems (Barbero, Vila y Suárez, 2006).

\section{Participantes}

La muestra elegida para el estudio empírico está constituida por estudiantes universitarios o ya egresados que accedieron voluntariamente a responder al cuestionario. La muestra utilizada está formada 288 personas, tamaño que se puede considerar suficiente atendiendo a los criterios que se exponen en Morales et al. (2003) para las escalas tipo Likert. En cuanto a su composición, de los 288 sujetos que respondieron al cuestionario, el 50.3\% son universitarios españoles y $49.7 \%$ universitarios mexicanos. La distribución de la misma en cuanto a género es tal que el $35.8 \%$ son varones y el $64.2 \%$ mujeres. La media de edad es de 22 años, con un valor mínimo de 18 y un valor máximo de 40. La distribución de la muestra según el tipo de estudios es la que se ofrece en el gráfico 1.

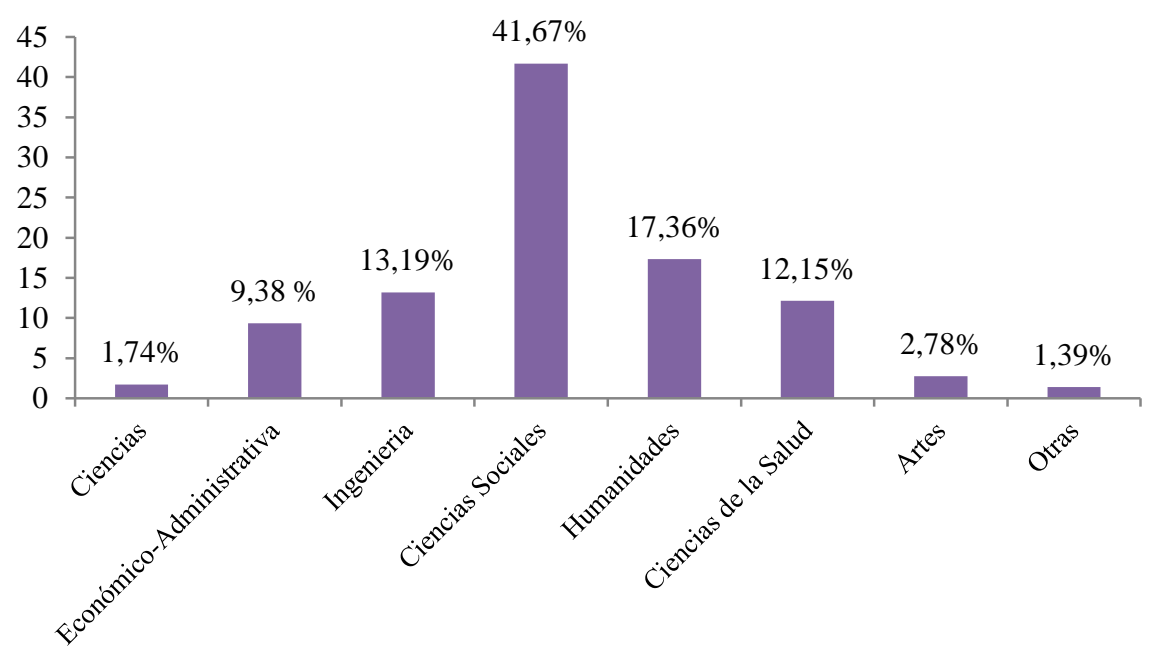

Gráfico 1. Distribución de la muestra por tipo de carrera universitaria.

La mayoría (el 96,9\%) son todavía estudiantes universitarios de los que solamente estudian (no trabajan) el 68,1\%. Del total de la muestra, el $19.8 \%$ no practica ni ha practicado deporte y el $80.2 \%$ sí realiza o ha realizado prácticas deportivas a distintos niveles (amateur el 76,8\% o profesional el 23,2\%). El tiempo semanal dedicado al deporte para el $25,2 \%$ de los practicantes es de 1 a 2 horas a la semana, mientras que para el $37 \%$ es de 2 a 8 horas y para el $37,8 \%$ restante es de más de 8 horas. Del total 
de la muestra el $37.2 \%$ practica deportes individuales y el $41.7 \%$ practica deportes por equipo. Solamente el $1,7 \%$ ha expresado practicar tanto deporte individual como colectivo.

\section{Resultados}

\section{Validez de contenido}

En el proceso de validación de contenido se procedió a solicitar a 21 expertos que emitieran su opinión de cada ítem en un formato diseñado para ese fin. Cada ítem fue calificado por su claridad, su especificidad y su significatividad (Castejón, 2006). Se utilizó una escala de 4 niveles, en la que el 1 representa el valor de máximo desacuerdo, el 2 de desacuerdo, el 3 de acuerdo y el 4 de máximo acuerdo. Una vez recogida la valoración de los expertos, se realizó la estadística descriptiva con el programa estadístico SPSS versión 15. Para el análisis de cada ítem se utilizó el siguiente criterio de evaluación:

- Si la media, mediana o moda resultó igual o menor a 3 y la desviación típica mayor a 9.5 los ítems fueron revisados.

- Si la media, mediana o moda tuvo valores menores o iguales a 2 los ítems fueron eliminados.

Como resultado del análisis estadístico y recomendaciones de los expertos, se obtiene la estructura final de cuestionario que se presenta en el anexo. La versión final del mismo está dividida en dos secciones:

- la primera sección se refiere a datos de identificación y clasificación y contiene 22 preguntas;

- en la segunda, se encuentra el núcleo del instrumento que consiste en un listado de 62 cuestiones sobre competencias genéricas. Este apartado se subidivide a su vez de modo que los primeros 20 elementos se refieren a competencias emocionales, los 40 siguientes a competencias gerenciales y otras competencias laborales. El ítem 61 es un elemento cuantitativo de referencia global al nivel competencial autopercibido y el ítem 62 es una pregunta abierta.

En esta segunda parte, se utiliza una escala de respuesta tipo likert graduada en 6 niveles, en la que el encuestado debe elegir, sabiendo que el 1 significa "nunca" y el 6 "siempre". La elección de un número par de respuestas se hizo pensando en sus ventajas, entre las cuales se ha valorado, especialmente, la eliminación de la posibilidad de que el sujeto que responde se evada tendiendo a elegir la respuesta central (Morales et al., 2003).

El cuestionario fue diseñado también en formato de página web para ser respondido electrónicamente y el tiempo de respuesta varió entre 13 y 20 minutos aproximadamente. 
Validación empírica: Fiabilidad y análisis de los ítems.

La fiabilidad del instrumento, considerado en su conjunto y entendida ésta como consistencia interna, es muy alta, como puede apreciarse en la tabla 1.

\begin{tabular}{cc}
\hline Alfa de Cronbach & Número de elementos \\
\hline .958 & 60 \\
\hline Tabla 1. Coeficiente de fiabilidad para la prueba total
\end{tabular}

Tabla 1. Coeficiente de fiabilidad para la prueba total

El índice de homogeneidad (IH) de los ítems resulta siempre positivo (ver gráfico 2), con un valor promedio de 0,54 , alcanza un valor máximo de 0.684 (para el ítem 44) y un valor mínimo de 0.112 (para el ítem 60). El ítem 4 tiene una correlación con la escala corregida de 0,299 y para todos los demás el IH obtenido supera el valor 0,3. El 60 sería pues el único ítem con cuya eliminación mejoraría la fiabilidad total de la prueba, que pasaría a ser de 0,96 .

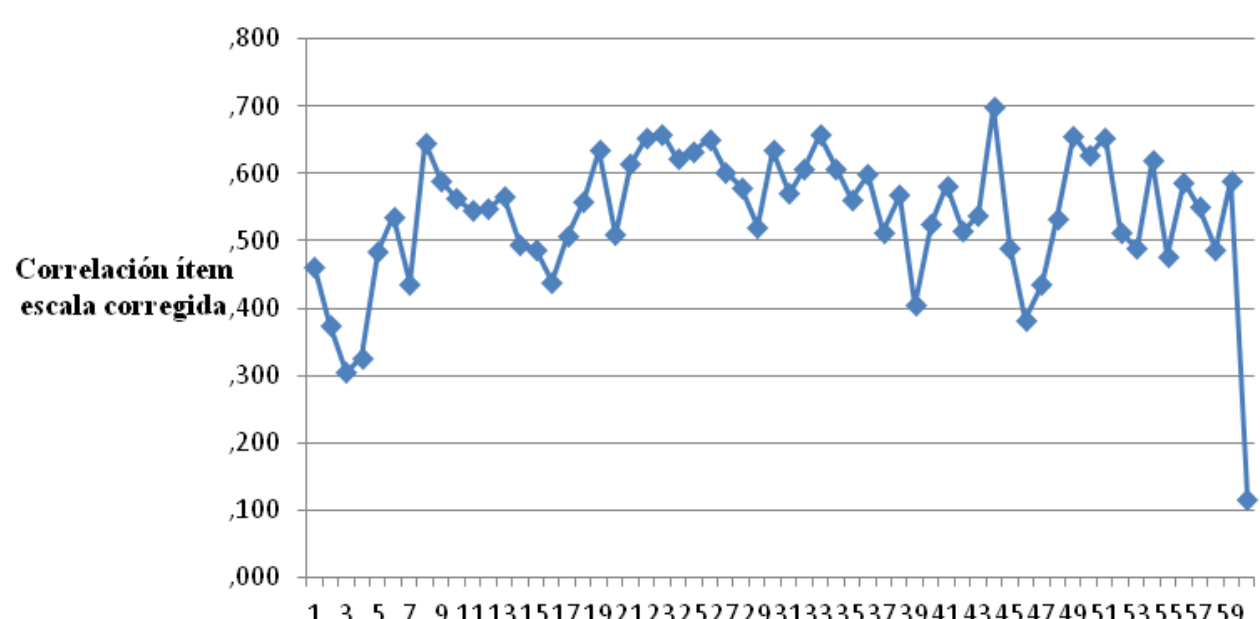

Número de Pregunta

Gráfico 2. Índices de homogeneidad de los ítems.

La correlación de cada item con el total de la escala, eliminado el ítem que se analiza, aparte de ser un indicativo de la fiabilidad del mismo, aporta además información acerca de la capacidad de discriminación que tiene, de ahí que en la bibliografía especializada también reciba el nombre de "índice de discriminación" (ID) (Muñiz et al., 2005). La discriminación de los items puede evaluarse además estudiando las diferencias en la puntuación total entre los grupos extremos: el $25 \%$ de los sujetos que tienen la puntuación más baja en la escala (por debajo del centil 25 que es 4,3167 ) y el $25 \%$ de los que tienen la más alta (por encima del centil 75 , esto es, 5,0167). Los resultados que se obtienen para los ítems del 1 al 59 cuando se aplica este 
procedimiento son siempre significativos, con probabilidades cero o muy cercanas a cero, a favor siempre del grupo superior. En el caso del elemento 60, la $t$ de Student obtenida ha sido de $-1,933$ ( $\mathrm{p}=0,056$ ), esto es, a favor del grupo 2 pero no significativa. Con ello se puede concluir que todos los ítems, salvo el número 60, discriminan adecuadamente entre grupos extremos, lo que, como era de esperar, está en consonancia con las conclusiones que se derivan de los IH obtenidos anteriormente.

El estudio de la fiabilidad empírica del instrumento se puede abordar también considerando que éste está compuesto de dos subescalas: por un lado las competencias emocionales, cuya estructura ha sido estudiada desde la investigación sobre inteligencia emocional, y por otro lado, otras competencias gerenciales y laborales que se suelen demandar desde múltiples campos profesionales. Con este planteamiento los coefientes encontrados resultan también altamente satisfactorios, como se observa en las tablas 2 y 3 .

\begin{tabular}{cc}
\hline Alfa de Cronbach & No. de elementos \\
\hline Tabla 2. Coeficiente de fiabilidad para las competencias emocionales \\
\hline Alfa de Cronbach \\
\hline Tabla 3. Coeficiente de fiabilidad para las competencias gerenciales y otras \\
competencias laborales.
\end{tabular}

Que la fiabilidad disminuya es natural puesto que disminuye el número de ítems, pero en cualquier caso, los coeficientes obtenidos indican una alta consistencia interna. En las Tablas 4 y 5 se presenta, para cada ítem, su $\mathrm{IH}$, su desviación típica $\left(\mathrm{S}_{\mathrm{i}}\right)$ y el índice de fiabilidad (IF, obtenido de multiplicar la correlación de cada ítem con la escala corregida eliminando el ítem que se analiza por la desviación típica del ítem en cuestión) (Barbero et al., 2006). En la última columna se ofrece el cambio en la fiabilidad total de la escala que supondría la eliminación del ítem, de lo que se desprende que la supresión de cualquier ítem llevaría asociada una pérdida, aunque mínima, en la fiabilidad total, excepción hecha del ítem 60, que es el único, otra vez, cuya eliminación supondría una mejora en el coeficiente de fiabilidad de la medida de las competencias gerenciales y laborales. 


\begin{tabular}{crrrr}
\hline Elemento & IH & S & IF & $\begin{array}{c}\text { Alfa de Cronbach si se eliminara } \\
\text { el elemento }\end{array}$ \\
& & & &, 879 \\
1 &, 465 & 1,035 & 0,48 &, 881 \\
3 &, 392 & 1,083 & 0,42 &, 883 \\
4 &, 326 & 0,934 & 0,30 &, 882 \\
5 &, 334 & 0,901 & 0,30 &, 877 \\
6 &, 508 & 0,87 & 0,44 &, 876 \\
7 &, 537 & 1,024 & 0,55 &, 880 \\
8 &, 454 & 1,255 & 0,57 &, 872 \\
9 &, 658 & 1,014 & 0,67 &, 875 \\
10 &, 579 & 0,955 & 0,55 &, 875 \\
11 &, 570 & 1,097 & 0,63 &, 877 \\
12 &, 506 & 1,198 & 0,61 &, 877 \\
13 &, 518 & 0,876 & 0,45 &, 875 \\
14 &, 576 & 1,031 & 0,59 &, 879 \\
15 &, 512 & 0,857 & 0,44 &, 879 \\
16 &, 462 & 0,989 & 0,46 &, 877 \\
17 &, 457 & 0,899 & 0,41 &, 877 \\
18 &, 526 & 0,889 & 0,47 &, 876 \\
19 &, 520 & 0,918 & 0,48 &, 880 \\
20 &, 551 & 0,99 & 0,55 & 0,45 \\
\hline
\end{tabular}

Tabla 4. Índices de homogeneidad y fiabilidad de los ítems que miden competencias emocionales

\begin{tabular}{crrrr}
\hline Elemento & IH & \multicolumn{1}{c}{$\mathrm{S}_{\mathrm{i}}$} & IF & Alfa de Cronbach si se eliminara el elemento \\
& & & &, 943 \\
21 &, 559 & 1,042 & 0,58 &, 942 \\
22 &, 642 & 1,09 & 0,70 &, 942 \\
23 &, 632 & 0,983 & 0,62 &, 943 \\
24 &, 582 & 1,142 & 0,66 &, 943 \\
25 &, 614 & 1,052 & 0,65 &, 942 \\
26 &, 638 & 1,072 & 0,68 &, 943 \\
27 &, 589 & 1,047 & 0,62 &, 943 \\
28 &, 560 & 0,897 & 0,50 &, 943 \\
29 &, 488 & 0,856 & 0,42 &, 943 \\
30 &, 609 & 1,053 & 0,64 &, 943 \\
31 &, 570 & 0,972 & 0,55 &, 943 \\
32 &, 592 & 0,962 & 0,57 &, 942 \\
33 &, 658 & 0,895 & 0,59 &, 943 \\
34 &, 621 & 0,902 & 0,56 &, 943 \\
35 &, 574 & 0,944 & 0,54 &, 943 \\
36 &, 605 & 0,894 & 0,54 &, 944 \\
37 &, 471 & 0,99 & 0,47 &, 943 \\
38 &, 547 & 1,064 & 0,58 &, 944 \\
39 &, 379 & 0,998 & 0,38 &
\end{tabular}




$\begin{array}{lllll}40 & , 488 & 1,079 & 0,53 & , 943 \\ 41 & , 578 & 0,953 & 0,55 & , 943 \\ 42 & , 494 & 0,997 & 0,49 & , 943 \\ 43 & , 505 & 0,851 & 0,43 & , 943 \\ 44 & , 685 & 0,931 & 0,64 & , 942 \\ 45 & , 487 & 0,996 & 0,49 & , 943 \\ 46 & , 376 & 0,958 & 0,36 & , 944 \\ 47 & , 409 & 1,101 & 0,45 & , 944 \\ 48 & , 557 & 0,907 & 0,51 & , 943 \\ 49 & , 626 & 1,023 & 0,64 & , 942 \\ 50 & , 595 & 1,057 & 0,63 & , 943 \\ 51 & , 637 & 1,016 & 0,65 & , 942 \\ 52 & , 490 & 1,078 & 0,53 & , 943 \\ 53 & , 471 & 1,119 & 0,53 & , 944 \\ 54 & , 604 & 1,111 & 0,67 & , 943 \\ 55 & , 456 & 1,495 & 0,68 & , 944 \\ 56 & , 579 & 1,156 & 0,67 & , 943 \\ 57 & , 536 & 1,207 & 0,65 & , 944 \\ 58 & , 486 & 1,065 & 0,52 & , 943 \\ 59 & , 596 & 0,927 & 0,55 & , 948 \\ 60 & , 124 & 1,544 & 0,19 & \end{array}$

Tabla 5. Índices homogenedidad y fiabilidad de los ítems que miden competencias gerenciales y otras competencias laborales

Para el estudio de aproximación a la validez concurrente de los ítems, el ítem considerado como criterio es el 61, en el que se pregunta, con un grado máximo de inferencia, acerca del nivel competencial general autopercibido. La correlación de cada ítem con el criterio muestra un valor promedio de 0.344 , con un máximo de 0.508 y un mínimo de -0.043 , en un rango promedio de 0.236 a 0.413 (ver gráfico 3 ).

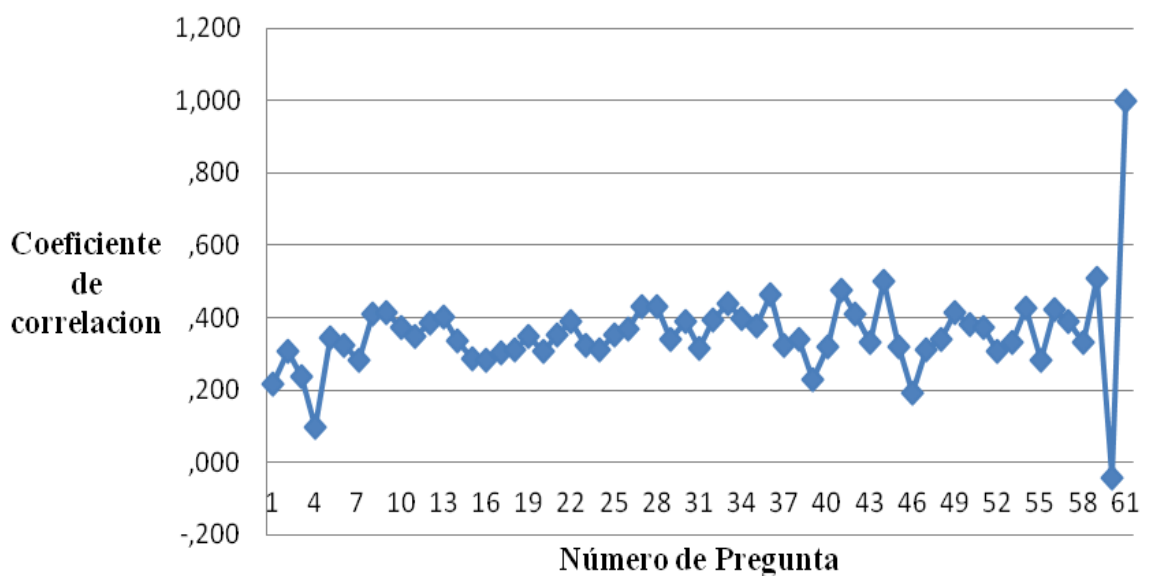

Gráfico 3. Correlación de cada ítem con el ítem criterio. 
Los ítems que no alcanzaron un indice de validez (IV, producto de la desviación típica del ítem por la correlación entre el ítem que se analiza y el criterio) (Barbero et al., 2006) adecuado son el 4 y el 60 (Tabla 6). Para el resto, la correlación con el criterio es significativa con un $\alpha=0.01$.

\begin{tabular}{ccccc}
\hline & Ítem 44 & Ítem 59 & Ítem 4 & Ítem 60 \\
\hline $\begin{array}{c}\text { Correlación } \\
\text { item-criterio }\end{array}$ & $\begin{array}{c}0.502 \\
* *\end{array}$ & $\begin{array}{c}0.508 \\
* *\end{array}$ & 0.096 & -0.043 \\
\hline Sig. & 0.000 & 0.000 & 0,10 & 0.466 \\
\hline IV & 0,473 & 0,471 & 0,086 & $-0,066$ \\
\hline
\end{tabular}

Tabla 6. Valores máximos y mínimos para el índice de validez (IV)

Para el estudio de la utilidad de la prueba, se ha analizado, por último, el impacto de los items en diferentes grupos de referencia que pueden llegar a ser de interés en el marco de una investigación más amplia. Se ha probado así la capacidad de los ítems para discriminar entre hombres y mujeres, entre mexicanos y españoles, entre ramas universitarias y, con especial interés, entre deportistas y no deportistas. En la Tabla 7 se ofrece el resumen de los análisis realizados (sólo figuran los valores del estadístico con su probabilidad asociada cuando ha resultado significativo).

\begin{tabular}{|c|c|c|c|c|c|c|c|c|}
\hline \multirow{2}{*}{ Elemento } & \multicolumn{2}{|c|}{ Sexo } & \multicolumn{2}{c|}{ País } & \multicolumn{2}{c|}{ Carrera } & \multicolumn{2}{c|}{ Práctica de deporte } \\
\hline & $\mathrm{F}_{(1,286)}$ & Sign. & $\mathrm{F}_{(1,286)}$ & Sign. & $\mathrm{F}_{(7,279)}$ & Sign. & $\mathrm{F}_{(1,286)}$ & Sign. \\
\hline 1 & 27,664 &, 000 & 32,712 &, 000 & 5,642 &, 000 & 18,372 &, 000 \\
\hline 2 & & & & & & & 7,599 &, 006 \\
\hline 3 & & & & & & & 4,527 &, 034 \\
\hline 4 & & & & & & & 4,994 &, 026 \\
\hline 5 & 4,760 &, 030 & 8,013 &, 005 & & & 9,775 &, 002 \\
\hline 6 & 14,610 &, 000 & 23,144 &, 000 & 2,457 &, 018 & 23,689 &, 000 \\
\hline 7 & 24,558 &, 000 & 10,995 &, 001 & 2,934 &, 006 & 7,045 &, 008 \\
\hline 8 & 19,041 &, 000 & 26,873 &, 000 & 3,240 &, 003 & 31,697 &, 000 \\
\hline 9 & & & 31,215 &, 000 & 3,060 &, 004 & 21,198 &, 000 \\
\hline 10 & 5,740 &, 017 & 27,281 &, 000 & & & 28,568 &, 000 \\
\hline 11 & & & 58,925 &, 000 & 3,641 &, 001 & 25,165 &, 000 \\
\hline 12 & & & 6,670 &, 010 & 2,135 &, 040 & 4,888 &, 028 \\
\hline 13 & 9,491 &, 002 & 17,628 &, 000 & & & 11,752 &, 001 \\
\hline 14 & & & & & & & 5,950 &, 015 \\
\hline 15 & & & 4,116 &, 043 & & & & \\
\hline 16 & & & & & & & & \\
\hline 17 & 3,873 &, 050 & & & & & & \\
\hline 18 & & & & & & & & \\
\hline 19 & & & & & & & & \\
\hline 20 & & & & & 2,066 &, 047 & & \\
\hline 21 & & & 12,343 &, 001 & 2,645 &, 012 & 4,782 &, 030 \\
\hline 22 & 7,426 &, 007 & 24,267 &, 000 & 4,294 &, 000 & 6,227 &, 013 \\
\hline
\end{tabular}




\begin{tabular}{|c|c|c|c|c|c|c|c|c|}
\hline 23 & 5,585 & ,019 & 30,361 & ,000 & 5,723 & ,000 & 13,182 & ,000 \\
\hline 24 & 15,506 & ,000 & 30,725 & ,000 & 3,733 & ,001 & 23,308 &, 000 \\
\hline 25 & 4,485 & ,035 & 43,359 & ,000 & 3,628 & 001 & 22,534 & ,000 \\
\hline 26 & & & 12,453 &, 000 & & & 8,881 &, 003 \\
\hline 27 & & & 18,773 & ,000 & 2,568 & 014 & 9,488 & ,002 \\
\hline 28 & & & & & 4,721 & ,000 & 5,762 & 017 \\
\hline 29 & & & & & 2,568 &, 014 & & \\
\hline 30 & & & & & & & 5,976 &, 015 \\
\hline \multicolumn{9}{|l|}{31} \\
\hline 32 & & & & & & & 3,941 & 0,048 \\
\hline 33 & & & & & & & 6,079 & ,014 \\
\hline 34 & & & & & 2,092 & 044 & & \\
\hline 35 & & & & & 3,215 &, 003 & & \\
\hline \multicolumn{9}{|l|}{36} \\
\hline 37 & 4,126 & ,043 & 16,390 & ,000 & 2,475 & ,018 & 5,679 & ,018 \\
\hline 38 & & & 11,087 & ,001 & & & 4,802 &, 029 \\
\hline 39 & & & & & & & 13,212 & ,000 \\
\hline 40 & & & & & & & 5,367 & ,021 \\
\hline \multicolumn{9}{|l|}{41} \\
\hline 42 & & & & & 2,139 & ,040 & & \\
\hline 43 & & & & & 2,371 & 023 & & \\
\hline 44 & & & 14,805 & ,000 & 3,940 & ,000 & 22,500 & ,000 \\
\hline \multicolumn{9}{|l|}{45} \\
\hline 46 & 5,394 & ,021 & 12,580 & ,000 & 3,005 & ,005 & & \\
\hline 47 & & & 8,591 & ,004 & & & & \\
\hline 48 & & & & & 2,778 & ,008 & & \\
\hline 49 & 10,896 & ,001 & 25,296 & ,000 & 3,077 & ,004 & 30,425 & 000 \\
\hline 50 & & & 19,516 & ,000 & 2,754 & ,009 & 21,734 & ,000 \\
\hline 51 & 9,881 &, 002 & 18,510 & 000 & 2,796 & ,008 & 10,516 & ,001 \\
\hline 52 & 10,754 & ,001 & 20,576 & ,000 & 3,068 & ,004 & 4,185 &, 042 \\
\hline 53 & & & 11,524 & ,001 & 2,288 & ,028 & 4,085 & ,044 \\
\hline 54 & 5,863 & 016 & 26,018 & 000 & 3,671 & ,001 & 12,490 &, 000 \\
\hline 55 & 4,964 & ,027 & 56,982 & ,000 & 4,082 & ,000 & 35,810 &, 000 \\
\hline 56 & & & 38,750 & ,000 & 4,336 & ,000 & 24,718 & ,000 \\
\hline 57 & 17,053 & 000 & 25,824 & 000 & 3,947 & ,000 & 19,065 & ,000 \\
\hline 58 & 15,768 & ,000 & 18,048 & ,000 & 3,239 & ,003 & & \\
\hline 59 & & & & & & & 4,931 & 027 \\
\hline 60 & 7,634 & ,006 & 14,549 & ,000 & 3,092 & ,004 & & \\
\hline 61 & 4,347 & ,038 & 8,383 & ,004 & & & $\begin{array}{c}17,039 \\
\end{array}$ &, 000 \\
\hline
\end{tabular}

Tabla 7. Impacto de los ítems por sexo, país, carrera, práctica de deporte (sí o no) 


\section{Conclusiones}

El trabajo realizado supone un apoyo empírico a la validez de la medida objeto de estudio, puesto que, en general, los coeficientes e índices obtenidos son satisfactorios. En lo referente a la fiabilidad, tanto la prueba en su conjunto como las dos subescalas existentes arrojan coeficientes altos y muy altos. En cuanto al análisis de los ítems que componen la escala, la mayoría de los índices conseguidos suponen un aval empírico a la calidad de los mismos cuando se evalúa tanto su fiabilidad, como su validez y capacidad discriminativa.

Los resultados obtenidos en el estudio empírico aquí resumido se presentan como extraordinariamente útiles para avanzar en la depuración del instrumento competest, el cual, como medida global de las competencias genéricas seleccionadas, ha evidenciado tener una consistencia interna alta, unos ítems claramente homogéneos, a excepción hecha del elemento 60, y válidos para la medida del nivel competencial globalmente percibido, salvo en el caso de los elementos 4 y 60, dos únicos ítems con valores de validez concurrente no satisfactorios

Del estudio realizado se desprende que del total de ítems analizados, la mayoría aportan diferente nivel de impacto para los grupos que se derivan de las variables de clasificación elegidas, siendo la variable para la que se encuentran más diferencias significativas el practicar o no deporte. En este estudio la práctica de deporte aparece pues como una variable relevante que se asocia con la mayoría de los ítems que miden competencias emocionales y otras competencias directivas y laborales, lo cual da idea del impacto que dicha variable tiene en la autopercepción de las competencias genéricas estudiadas.

Los elementos 16, 18, 19, 31, 36, 41 y 45 no discriminan entre los grupos en ninguna de las variables elegidas, sin embargo, todos ellos son valiosos porque aportan tanto a la fiabilidad como a la validez concurrente de la medida y discriminan entre los grupos del $25 \%$ superior y el $25 \%$ inferior según la puntuación media total en el cuestionario. El ítem 60 es el único que ha obtenido en el estudio piloto realizado unos índices de fiabilidad y validez insuficientes y tampoco discrimina entre practicantes y no practicantes de deporte.

Se plantea como prospectiva de investigación conseguir una medida más parsimoniosa y depurada, a partir de la reflexión acerca de la inclusión de preguntas con correlaciones excesivamente altas con el total de la prueba, y la validación de constructo, que se habrá de abordar mediante la utilización de técnicas como el Análisis Factorial Exploratorio y Confirmatorio

La medida de las competencias genéricas autopercibidas se presenta como un instrumento útil de cara al diagnóstico y evaluación en la universidad, donde la herramienta competest se puede usar con fines formativos e informativos del proceso de aprendizaje, valioso para profesores y para los propios estudiantes, y con fines sumativos, como complemento de otras técnicas, para calificar al estudiante considerando no sólo el grado de dominio de las competencias específicas, sino 
además el nivel de incorporación de los saberes teóricos y prácticos en su saber ser como persona, ciudadano y profesional.

El análisis de los ítems que componen el cuestionario pone de manifiesto su utilidad especial como herramienta para medir el impacto de la práctica del deporte en la autopercepción de determinadas competencias genéricas comúmnente valoradas como necesarias para el desempeño profesional de calidad y, por tanto, como instrumento de investigación sobre la relación entre la práctica deportiva, en sus diferentes niveles y modalidades, y el desarrollo competencial autopercibido. En este sentido, puede llegar a ser una medida muy útil para el estudio de la relación entre determinadas prácticas deportivas y la formación o desarrollo de competencias genéricas que en un momento dado pueda llegar a plantearse desarrollar en estudios experimentales concretos, tanto en el ámbito de la educación general a nivel superior, como en el ámbito del aprendizaje específico de algún perfil profesional o deportivo particular. Los resultados de esta línea de investigación pueden abrir vías para la utilización del deporte como acción instructiva intencional y estructurada con el fin de lograr el desarrollo de las competencias genéricas percibidas como más necesarias desde diferentes campos profesionales y en las que los programas convencionales de formación en la universidad actual aparecen como más deficitarios.

\section{Referencias bibliográficas}

ALLES, M. (2002/2006) Gestión por Competencias: El Diccionario. Buenos Aires: Granica.

ÁLVAREZ ROJO, V. (2011). Un recurso virtual de apoyo al profesorado para la evaluación de competencias. En EVALfor (Ed.), EVAL trends 2011 - Evaluar para aprender en la universidad: Experiencias innovadoras de evaluación en la era digital (pp.187-199). Madrid: Bubok Publishing.

ARRUZA, J. A., ARRIBAS, S., GONZÁLEZ, O., BALAGUÉ, G., ROMERO, S. \& RUIZ, L. M. (2005). Desarrollo y validación de una versión preliminar de la Escala de Competencia emocional en el deporte (ECE-D). Motricidad, European Journal of Human Movement 14, 151-163

ASIS, B. de. (2007) Competencias profesionales en la Formación Profesional. Madrid: Alianza Editorial.

BARBERO, M.I.; VILA, E. Y SUÁREZ, J.C. (2006) Psicometría. Madrid: UNED

BENEITONE, P. (2008). La convergencia de la educación superior en América Latina: El impacto del Proyecto Tuning. A-Mérika, 1(1), 12.

BENEITONE, P. et al. (Eds.). (2007). Informe Final del Proyecto Tuning América Latina: Reflexiones y perspectivas de la Educación Superior en América Latina. Bilbao: Universidad de Deusto 
BISQUERRA, R. (2003). Educación emocional y competencias básicas para la vida. Revista de Investigación Educativa (RIE), 21, 1, 7-43.

BISQUERRA, R. \& PÉREZ, E. N. (2007). Las Competencias Emocionales. Educación $X X 1,10,61-82$

BLANCO, P. A. (2007) Trabajadores Competentes; Introducción y reflexiones sobre la gestión de recursos humanos por competencias. España: ESIC.

BOYATZIS, R. (1982). The Competent Manager. N.Y.: Wiley and Sons.

CANO, E. (2005). Cómo mejorar las competencias de los docentes: Guía para la autoevaluación y el desarrollo del profesorado. Barcelona: Ed. Grao

CASTEJÓN, J. et al. (2006). Elaboración, Análisis e Interpretación de Encuestas, Cuestionarios y Escalas de Opinión. Alicante: Marfil

CASTRO, M. (2011) ¿Qué sabemos de la medida de las competencias? Características y problemas psicométricos en la evaluación de competencias. Bordón, 63 (1), 109123

CONFERENCE OF EUROPEAN MINISTERS RESPONSIBLE FOR HIGHER EDUCATION (2009). Communique of the Conference of European Ministers Responsible for Higher Education, Leuven and Louvain-la-Neuve: The Bologna Process 2020 - The European Higher Education Area in the new decade. Recuperado de: http://www.ond.vlaanderen.be/hogeronderwijs/Bologna/conference/documents/Leu ven Louvain-la-Neuve Communiqué April 2009.pdf en octubre de 2012.

CUBEIRO, J.C. \& GALLARDO, L. (2008). Liderazgo, Empresa y Deporte. Madrid: Lid.

DELORS, J. et al. (1998). La educación encierra un tesoro: Informe a la UNESCO de la Comisión Internacional sobre la Educación para el Siglo XXI. Madrid: Santillana.

EUROPEAN UNIVERSITY ASSOCIATION (2010). Trends 2010. Recuperado de: http://www.eua.be/fileadmin/user_upload/files/Publications/Trends_2010.pdfen enero de 2012.

FERNÁNDEZ BERROCAL, P. EXTREMERA, N., PALOMERA, R., RUIZARANDA, D., SALGUERO, J.M. Y CABELLO, R. (Eds.) (2009) Avances en el estudio de la Inteligencia Emocional. I Congreso Internacional de Inteligencia Emocional. Santander: Fundación Marcelino Botín.

GOLEMAN, D. (2007). La Práctica de la Inteligencia Emocional. 20ª Edición. Barcelona: Kairós.

GONZÁLEZ, J. \& WAGUENAAR, R. (Eds.) (2003). Tuning Educational Estructure in Europe; Informe final. Fase I. Bilbao: Universidad de Deusto. 
GONZÁLEZ, J. \& WAGUENAAR, R. (Eds.) (2006). Tuning Educational Estructure in Europe; Informe final. Fase II La contribución de las Universidades al Proceso de Bolonia. Bilbao: Universidad de Deusto.

GONZÁlEZ, J., WAGENAAR, R., \& BENEITONE, P. (2004). Tuning-América Latina: Un proyecto de las universidades. Revista Iberoamericana de Educación, 35, 151-164.

GONZÁLEZ, M.V. \& GONZÁLEZ, T. R. (2008). Competencias genéricas y formación profesional: Un análisis desde la docencia universitaria. Revista Iberoamericana de Educación, 47, 185- 209

HOLT, N. L. \& DUNN, J. H. (2004). Toward a Grounded Theory of the Psychosocial Competencies and Environmental Conditions Associated with Soccer Success. Journal Applied Sport Psychology. 16 (3), 199 - 219.

IBARRA, M. S. (dir.) (2008) EvalCOMIX: Evaluación de competencias en un contexto de aprendizaje mixto. Resultados del proyecto de Estudios y Análisis EA2007-0099 titulado "ProyectoEvalCOMIX: Evaluación de competencias en un contexto de aprendizaje mixto (Blended-Learning)" financiado por la Dirección General de Universidades del Ministerio de Educación y Ciencia. Resolución de 29 de mayo de 2007 (BOE141 de 13 de junio de 2007). Cádiz: Servicio de Publicaciones de la Universidad de Cádiz.

IGLESIAS, M. J. (2009). Elaboración y validación de un instrumento diagnóstico para la percepción de las competencias emocionales en estudiantes universitarios de educación. REOP, 20 (3), 330-311.

JONES, A. (2006). Professional skills and generic skills: context and culture; ICED Conference, Sheffield Hallam University, June.

MARTÍNEZ-RODRÍGUEZ, F. M. \& CARMONA, G. (2010). Test de factores sociopersonales para la inserción laboral de los jóvenes: Un instrumento para la evaluación y la formación. ESE Estudios sobre Educación, 18, 115-138.

MAYOCCHI, L. Y HANRAHAN, S., (2007). Transferable Skills for Career Change. En D. Lavallee \& P. Wylleman. Career Transitions in Sport. International Perspectives. Virginia University: Lavallee \& Wylleman Career editors, 96-110.

MENÉNDEZ, V. J. L. (2009). La noción de competencia en el proyecto Tuning. Un análisis textual desde la sociología de la educación. Observar. Revista Electrónica Del Observatorio sobre la Didáctica de las Artes, 3, 5-41

MERTENS, L. (1997/1996) “Competencia laboral: Sistemas, surgimiento y modelos". México: CINTERFOR (Centro Interamericano para el Desarrollo de Conocimiento en la Formación Profesional) / OIT (Organización Internacional del Trabajo).

MINISTER RESPONSIBLE FOR THE EUROPEAN HIGHER EDUCATION AREA (2010). Budapest-Vienna Declaration on the European Higher Education Area. 
Recuperado de: http://www.ond.vlaanderen.be/hogeronderwijs/Bologna/2010 conference/document s/Budapest-Vienna_Declaration.pdf en octubre de 2010,

MORALES, P., UROSA, B. Y BLANCO, A. (2003). Construcción de Escalas de Actitudes tipos Likert. Madrid: La Muralla.

MUÑIZ, J., FIDALGO, A.M., GARCÍA CUETO, E., MARTÍNEZ, R. Y MORENO, R. (2005). Análisis de los ítems. Madrid: La Muralla.

OIT (Organización Internacional del Trabajo) \& CINTERFORD (Centro Interamericano para el Desarrollo de Conocimiento en la Formación Profesional). (2010). Conceptos Básicos sobre Competencia Laboral. México: CINTERFORD/ OIT

ORDEN, A. DE LA. (2011) Educación y Competencias. Bordón, 63 (1), 9-12

ORDEN, A. DE LA., GARDUÑO, L., ASENSIO, I., MAFOKOZI, J., GONZÁLEZ, C., \& MUÑOZ, A. (2002). Funciones de la universidad. Revista de Educación, 328, 267-281.

PALMER, A., MONTAÑO, J. J. \& PALOU, M. (2009). Las competencias genéricas en la educación superior. Estudio comparativo entre la opinión de empleadores y académicos. Psicothema, 21 (3), 433-438

PEREDA, M.S. \& BERROCAL, B.F. (2001). Gestión de Recursos Humanos por Competencias. Madrid: Centro de Estudio Ramón Arce.

REPETTO, E. T., PENA, G. M., \& LOZANO, S. S. (2007) El Programa de Competencias socio-emocionales (POCOSE). XXI Revista de Educación, 9, 35-41

RODRÍGUEZ, E. A. \& VIEIRA, A. M. J. (2009). La Formación de Competencias en la Universidad: Un estudio empírico sobre su tipología. Revista de Investigación Educativa. 27 (1), $27-47$.

SAARNI, C. (2000). Emotional Competence; A Developmental Perspective. In R. Bar On y J. D.A. Parker (Eds.). The Handbook of Emotional Intelligence, 68-91. San Francisco: Jossey-Bass

SALOVEY, P. Y MAYER, J. D. (1990). Emotional Intelligence. Imagination, Cognition and Personality, 9,185-211

SALOVEY, P. Y MAYER, J. D. (1990). Emotional Intelligence. Massachusett: Baywood Publishing Co. Inc

SOLANES, A., NÚÑEZ, R. Y RODRÍGUEZ-MARÍN (2008). Elaboración de un cuestionario para la evaluación de competencias genéricas en estudiantes universitarios. Apuntes de Psicología, 26 (1), 35-49. 
STOUGH, SAKLOFKE, D.H. Y PARKER, J. D.A. (2009). Assessing Emotional Intelligence. Theory, Research, and Applications. London: Springer.

VILlA, A. \& POBLETE, M. (2007). Aprendizaje Basado en Competencias. Una propuesta para la evaluación de las competencias genéricas. Bilbao: Universidad de Deusto.

WEISINGER, A. (1998). La inteligencia emocional en el trabajo. Buenos Aires: Javier Vergara.

\section{Correspondencia con los autores}

I. Inmaculada ASENSIO MUÑOZ

Facultad de Educación Universidad Complutense de Madrid

C/ Rector Royo- Villanova, s/n. 28040 Madrid.

correo-e: macu@edu.ucm.es 


\section{ANEXO 1 \\ COMPETEST}

Este cuestionario ha sido diseñado para evaluar el grado de desarrollo de las competencias genéricas de las que haces uso en tus actividades diarias.

A continuación te pedimos, por favor respondas escogiendo solo una de las opciones que se proponen o aportando el dato.

Las respuestas son confidenciales y se usarán únicamente con fines de investigación.

\section{SECCIÓN I}

\section{GRACIAS POR TU COLABORACIÓN}

1.- Edad.

2.- Género

3.- Escribe el nombre de la universidad a la que perteneces o perteneciste.

4.- ¿Qué tipo de carrera estudiaste o estás estudiando?
(a) Humanidades
(b) Económico Administrativa
(d) Ciencias Sociales
(e) Artes
(f) Ciencias
(c) Ciencias de la Salud

5.- ¿Cuánto tiempo hace que has terminado tus estudios universitarios o actualmente estudias?

(a) Actualmente estudio (b) terminé hace menos de 3 años (c) terminé hace más de 3 y menos de 5 (d) terminé hace más de 5 años

6.- ¿Cuentas con postgrado?

7.- ¿Trabajas actualmente?

8.- ¿Cuál es tu actividad principal en la actualidad?

\begin{tabular}{|l|}
\hline si \\
\hline si \\
\hline
\end{tabular}

$\begin{array}{lll}\text { (a) Estudio } & \text { (b) Estudio y trabajo } & \text { (c) Tengo un empleo acorde a mi titulación universitaria }\end{array}$

Estoy realizando un trabajo por debajo de mi nivel de cualificación (e) Estoy buscando trabajo

dedico al deporte profesional (g) otros

9.-Indica por favor tu profesión.

10.- ¿Tienes algún puesto directivo?

En caso afirmativo, especifica cuál.

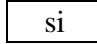

11.- ¿Actualmente cuánto tiempo llevas realizando la actividad que desempeñas?
(a) 1 años o menos
(b) 2 años
(c) 3 años o más

12.- ¿Practicas o has practicado algún deporte?

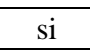

no

\section{(si tu respuesta es no, pasa directamente a la sección II}

13.- ¿Qué deporte practicas?

14.- ¿Desde cuándo practicas este deporte?
(a) desde antes de entrar en la Universidad
(b) comencé en la Universidad

(c) comencé cuando ya había terminado mis estudios universitarios

15.- ¿A qué nivel has practicado este deporte?
(a) amateur
(b) profesional

16.- ¿Cuánto tiempo le dedicas a la semana?
(a) una o dos horas
(b) tres o cuatro horas
(c) cinco a seis horas
(d) siete $\mathrm{u}$ ocho horas
(e) más de ocho horas
17.- ¿Representas o has representado a tu institución en competiciones?
18.- En caso afirmativo ¿En qué nivel de competición te presentaste?
(a) de invitación
(b) regional
(c) nacional
d) internacional

19.- ¿Qué lugar lograsteis en las competiciones?

(escribe la mejor posición lograda)

20.- ¿Qué tiempo has competido o llevas compitiendo?
(a) de 1 a 3 años
(b) de 3 a 5 años
(c) más de 5 años

21.- ¿Qué habilidades consideras haber desarrollado por el deporte?

22.- ¿Qué habilidades te resultan o te han resultado más útiles en tu actividad

como estudiante o como profesional? 


\section{SECCION II}

En esta sección te presentamos una escala de respuesta para que puedas contestar a cada pregunta de acuerdo a tu experiencia diaria señalando el cuadro que corresponda de 1 (nunca) a 6 (siempre)

Por lo general ¿con qué frecuencia...

\begin{tabular}{|c|c|c|c|c|c|c|c|}
\hline & & 1 & 2 & 3 & 4 & 5 & 6 \\
\hline 1 & tomas decisiones con seguridad sin dudar demasiado? & & & & & & \\
\hline 2 & actúas sin necesidad de dirección cuando hay imprevistos? & & & & & & \\
\hline 3 & reconoces tus diferentes estados de ánimo? & & & & & & \\
\hline 4 & identificas cuándo tu estado emocional no te favorece? & & & & & & \\
\hline 5 & tus decisiones son adecuadas ante situaciones adversas? & & & & & & \\
\hline 6 & mantienes un comportamiento estable ante situaciones de caos? & & & & & & \\
\hline 7 & $\begin{array}{l}\text { tratas de evitar que te desgasten física y emocionalmente las situaciones de } \\
\text { estrés? }\end{array}$ & & & & & & \\
\hline 8 & $\begin{array}{l}\text { tus acciones ayudan a superar más fácilmente un momento de tensión en tu } \\
\text { trabajo diario? }\end{array}$ & & & & & & \\
\hline 9 & $\begin{array}{l}\text { consideras que los objetivos planteados son alcanzables y vale la pena } \\
\text { intentarlo? }\end{array}$ & & & & & & \\
\hline 10 & $\begin{array}{l}\text { te muestras optimista hacia el logro de las metas incluso cuando tienes } \\
\text { malos resultados? }\end{array}$ & & & & & & \\
\hline 11 & buscas alcanzar metas más allá de las propuestas inicialmente? & & & & & & \\
\hline 12 & manifiestas interés en tu labor diaria? & & & & & & \\
\hline 13 & realizas comentarios oportunos en el momento preciso? & & & & & & \\
\hline 14 & tus acciones ayudan a mantener un buen clima de trabajo? & & & & & & \\
\hline 15 & detectas las necesidades de tus compañeros sin que ellos lo manifiesten? & & & & & & \\
\hline 16 & apoyas a tus compañeros cuando percibes que lo necesitan? & & & & & & \\
\hline 17 & escuchas atentamente la opinión de los demás para lograr acuerdos? & & & & & & \\
\hline 18 & ayudas a solucionar momentos de tensión o conflicto en el trabajo? & & & & & & \\
\hline 19 & interpretas las necesidades del grupo para compartirlas? & & & & & & \\
\hline 20 & intercambias con tus compañeros estrategias de trabajo? & & & & & & \\
\hline 21 & visualizas escenarios que favorezcan las metas a medio y largo plazo? & & & & & & \\
\hline 22 & te anticipas para afrontar exitosamente los retos futuros? & & & & & & \\
\hline 23 & detectas fácilmente oportunidades de desarrollo? & & & & & & \\
\hline 24 & tienes habilidad para transformar las debilidades en fortalezas? & & & & & & \\
\hline 25 & influyes en tus compañeros con el fin de obtener los mejores resultados? & & & & & & \\
\hline 26 & $\begin{array}{l}\text { fomentas con tus compañeros el logro de las metas en los momentos de } \\
\text { crisis? }\end{array}$ & & & & & & \\
\hline 27 & te sientes identificado con tu quehacer diario? & & & & & & \\
\hline 28 & te comprometes a realizar bien tu trabajo? & & & & & & \\
\hline 29 & te integras con tus compañeros para trabajar en forma colectiva? & & & & & & \\
\hline 30 & colaboras con tu equipo para impulsar ideas innovadoras? & & & & & & \\
\hline 31 & participas para complementar el trabajo en equipo? & & & & & & \\
\hline 32 & trabajas buscando el equilibrio del equipo? & & & & & & \\
\hline 33 & impulsas el trabajo hacia el logro de las metas? & & & & & & \\
\hline 34 & $\begin{array}{l}\text { aportas soluciones a los problemas que van surgiendo con el fin de alcanzar } \\
\text { los objetivos? }\end{array}$ & & & & & & \\
\hline 35 & te esfuerzas para cumplir en los tiempos establecidos? & & & & & & \\
\hline 36 & buscas hacer eficientes los recursos materiales para alcanzar las metas? & & & & & & \\
\hline 37 & expresas con claridad tus ideas? & & & & & & \\
\hline 38 & expresas tus ideas de forma convincente? & & & & & & \\
\hline 39 & detectas los mensajes no verbales de tus compañeros de trabajo? & & & & & & \\
\hline 40 & promueves los mensajes no verbales que favorecen el clima de trabajo? & & & & & & \\
\hline
\end{tabular}




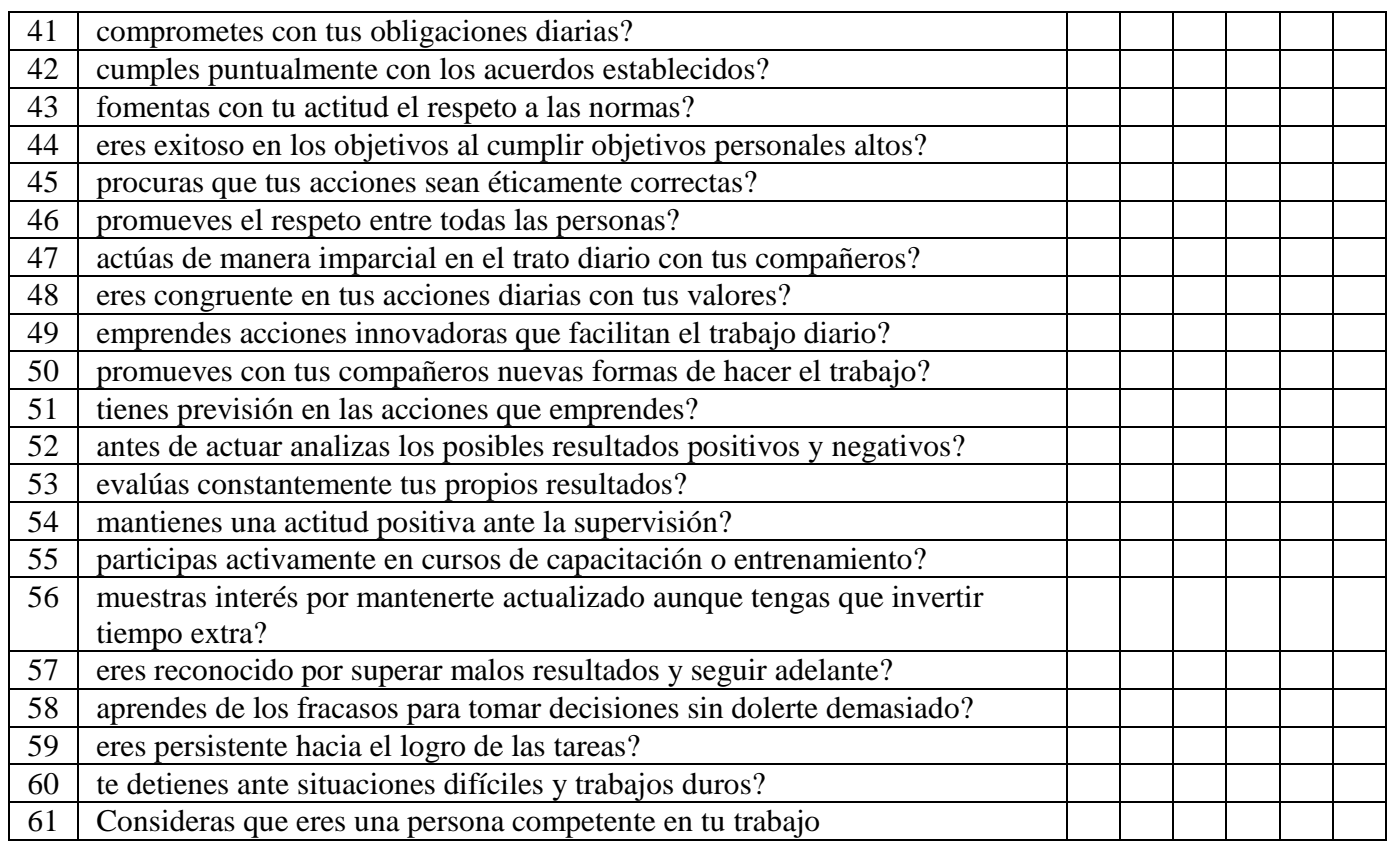

62 ¿Cómo consideras que has adquirido las habilidades que actualmente te exige tu trabajo? 